\title{
Quantitative Conformational Analysis of Functionally Important Electrostatic Interactions in the Intrinsically Disordered Region of Delta Subunit of Bacterial RNA Polymerase
}

Vojtěch Kubáń, ${ }^{\dagger, \ddagger}$ Pavel Srb, ${ }^{\ddagger}, \mathbb{q}$ Hana Štégnerová, ${ }^{\dagger}$ Petr Padrta, ${ }^{\ddagger}$ Milan Zachrdlaa, ${ }^{\dagger, \ddagger}$ Zuzana Jaseňáková, ${ }^{\dagger, \ddagger}$ Hana Šanderová, ${ }^{\S}$ Dragana Vítovská, ${ }^{\S}$ Libor Krásný, ${ }^{\S}$ Tomáš Koval',,$\|$ Jan

Dohnálek,, Joanna Ziemska-Legięcka, ${ }^{\perp}$ Marcin Grynberg, ${ }^{\perp}$ Patryk Jarnot, $\#$ Aleksandra Gruca,\# Malene Ringkjøbing Jensen, ${ }^{@}$ Martin Blackledge, ${ }^{* @}$ and Lukáš Žídek ${ }^{*, \dagger, \ddagger}$

$\dagger$ Masaryk University, Faculty of Science, National Centre for Biomolecular Research, Kamenice 5, 62500 Brno, Czech Republic

$\ddagger$ Central European Institute of Technology, Masaryk University, Kamenice 5, 62500 Brno, Czech Republic

IInstitute of Organic Chemistry and Biochemistry, Czech Academy of Sciences, v.v.i., Flemingovo nám 2, 16610 Prague 6, Czech Republic

$\S$ Laboratory of Microbial Genetics and Gene Expression, Institute of Microbiology of the Czech Academy of Sciences, v. v. i, Vídeňská 1083, 14220 Prague 4, Czech Republic

||Laboratory of Structure and Function of Biomolecules, Institute of Biotechnology of the Czech Academy of Sciences, v. v. i., Biocev, Prümyslová 595, 25250 Vestec, Czech Republic $\perp$ Institute of Biochemistry and Biophysics PAS, Pawinskiego 5A, 02-106 Warsaw, Poland \#Institute of Informatics, Silesian University of Technology, Akademicka 16, 44-100 Gliwice,

\section{Poland}

@ CEA, CNRS, and UJF-Grenoble, Protein Dynamics and Flexibility, Institut de Biologie Structurale Jean-Pierre Ebel, 71 avenue des Martyrs, Grenoble 38044, France

E-mail: martin.blackledge@ibs.fr; Izidek@chemi.muni.cz Phone: +33 457428 554; +420 549498393 

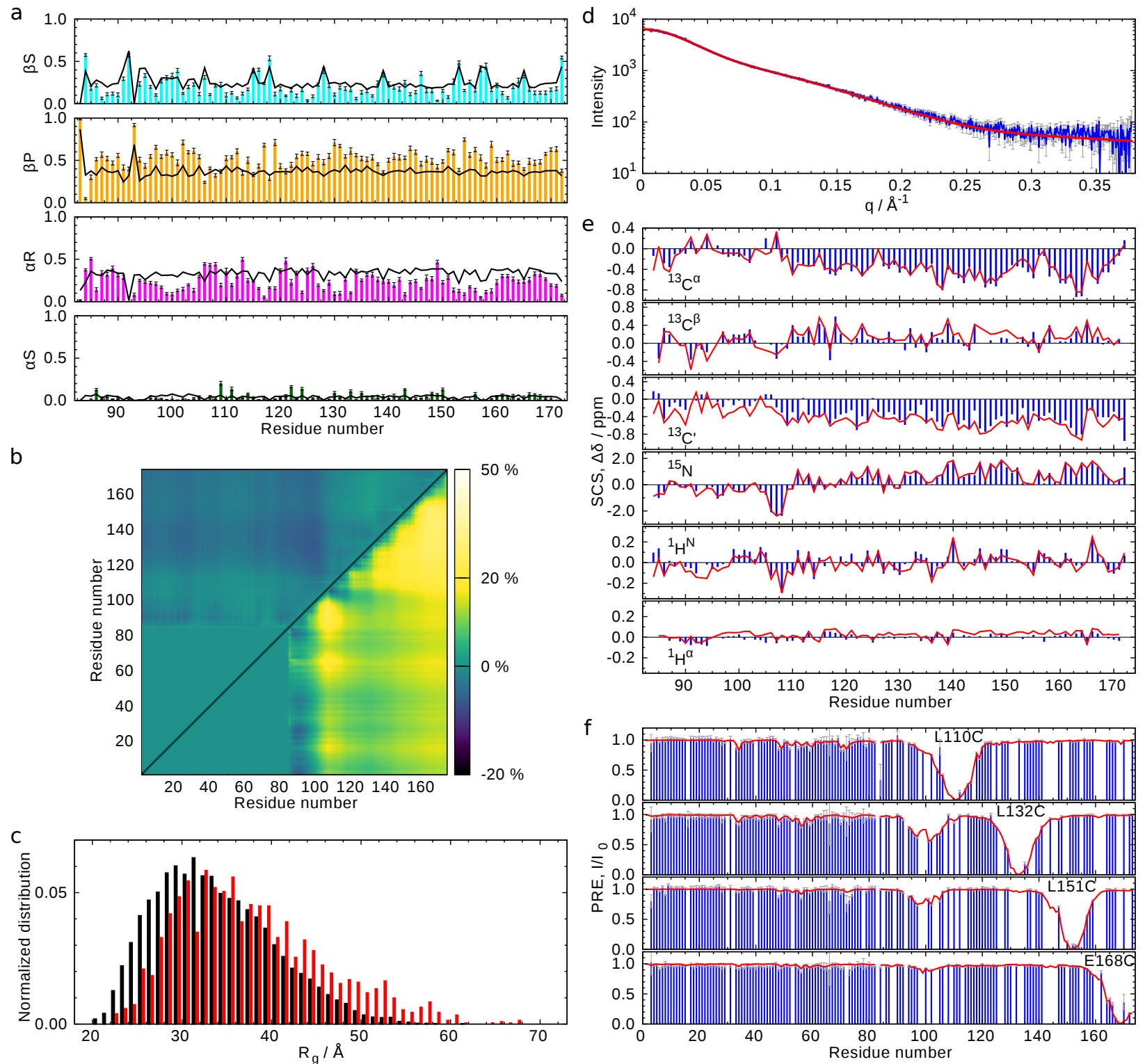

Supporting Figure S1: Conformational analysis of the $\delta$ subunit. (a) Populations in the selected ensembles were divided into regions in the Ramachandran diagrams, $\beta$ sheet (cyan), polyproline II (orange), $\alpha$ helix (magenta), and the rest with left-handed helix (green). The statistical-coil pool distribution in the Ramachandran diagram is black line. (b) Relative averaged deviations of mutual distances of $\mathrm{C}^{\alpha}$ (in \%) in the selected ensembles from the distances in the chemical-shift filtered (above diagonal) and the statistical-coil (below diagonal). Yellow and blue indicate longer and shorter distances, respectively. (c) Normalized distribution of radius of gyration for the selected ensembles (red) and the statistical-coil (black). The results were calculated by ASTEROIDS (red) based on the experimental data (blue) of (d) SAXS, (e) SCS, and (f) PRE. Ten ensembles with 200 conformers were calculated. 


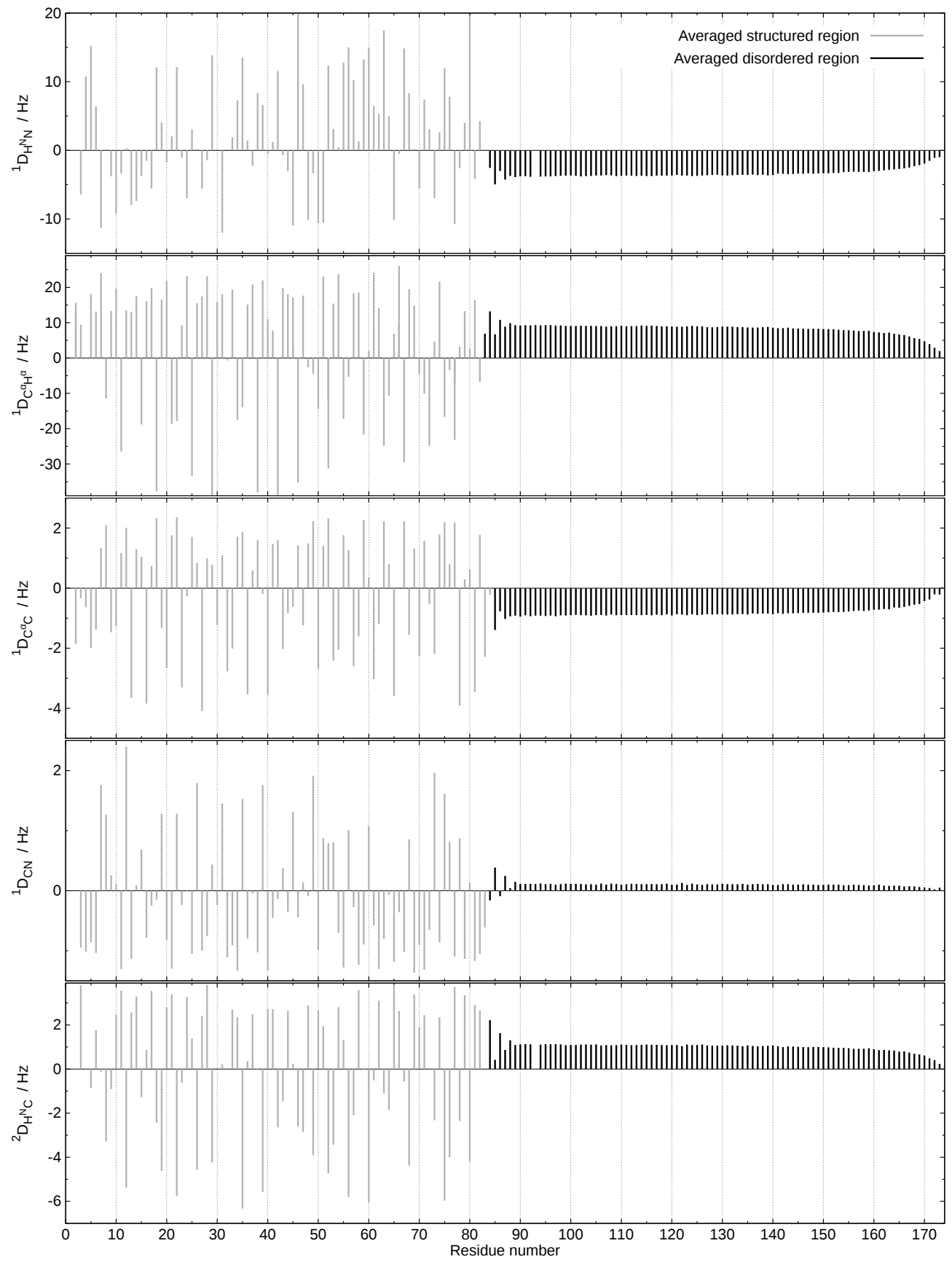

Supporting Figure S2: A global trend for RDC of the disordered C-terminus $\delta$ was obtained from an averaged RDCs of 250000 conformers. Conformers contain the structured region (gray) with substituted disordered region to polyvaline chain (black). Conformers were created by Flexible-Meccano. 


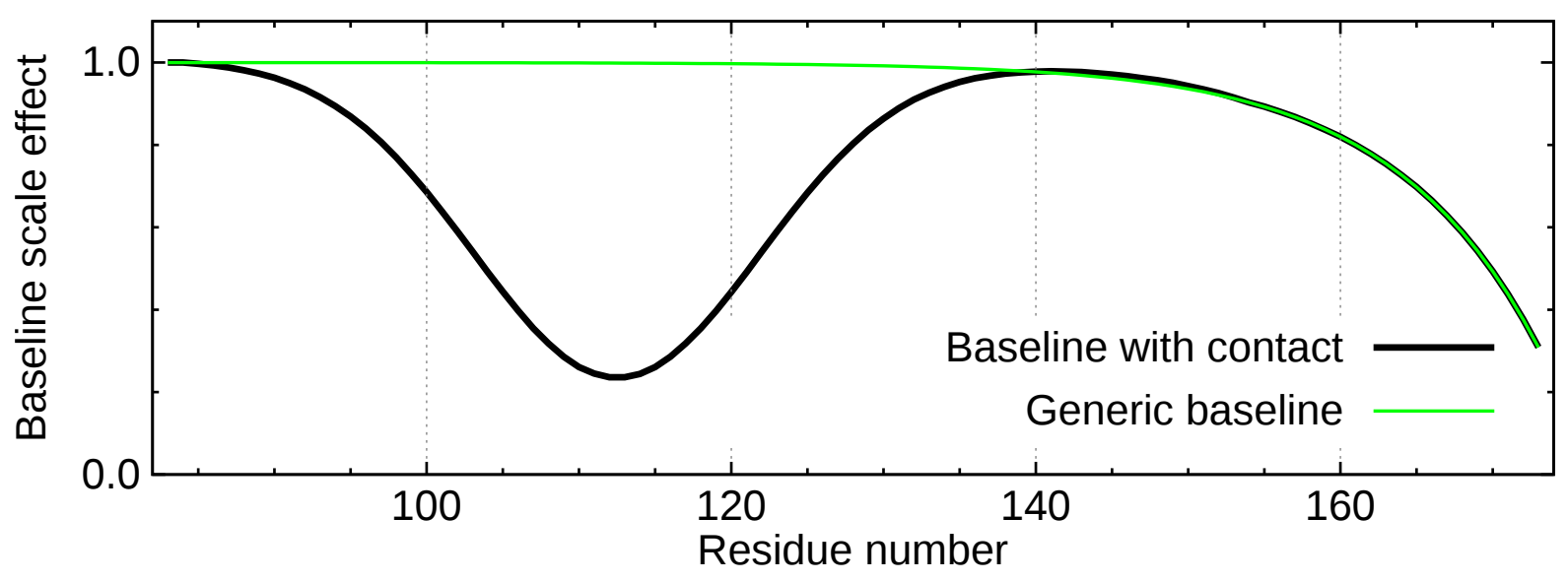

Supporting Figure S3: Baselines derived from polyvaline chain was used for $\delta_{\mathrm{KE}}$ (green). Contact between residues 95 and 130 was added into baseline for $\delta$ (black) based on the PRE experimental data. 

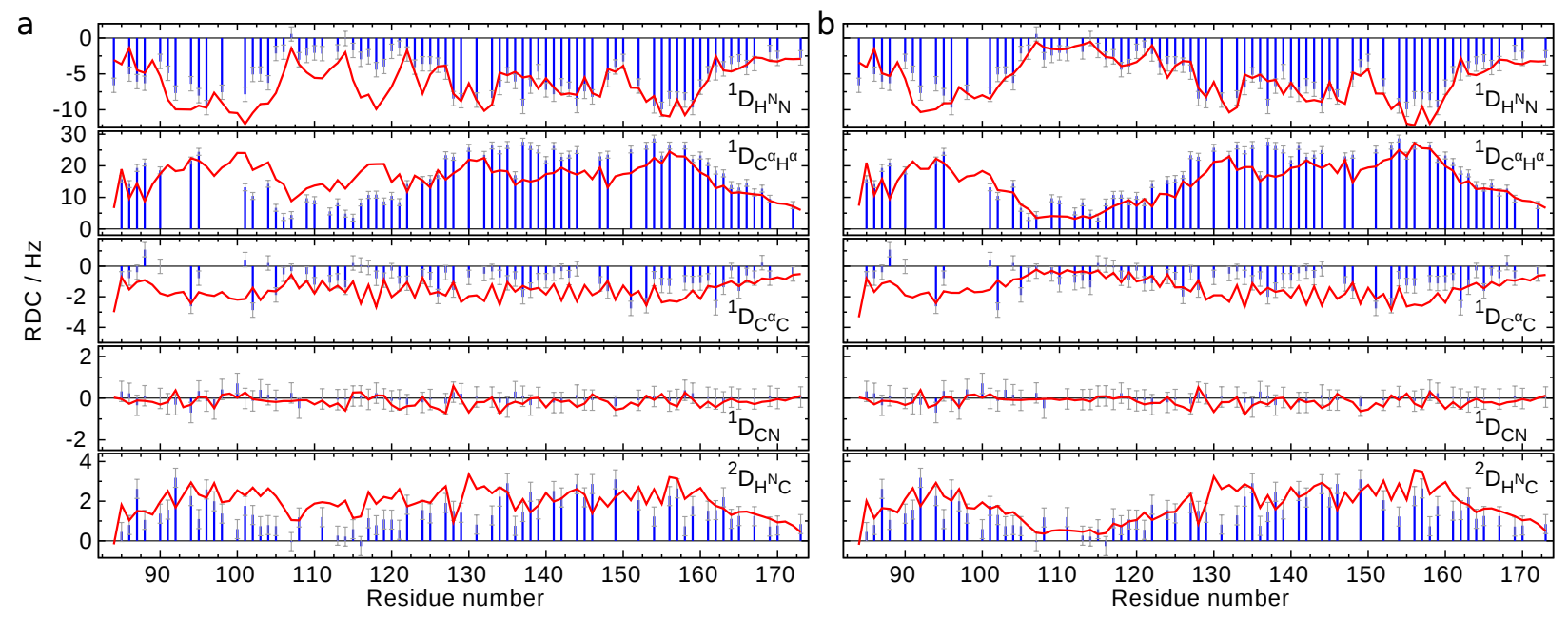

Supporting Figure S4: Cross-validation of RDC experimental data (blue) against predicted RDC from the selected ensemble presented in the Supporting Fig. 1 (red). (a) The half of the bell-shaped baseline was applied, whereas the baseline with the imposed contact was applied in (b). 
a

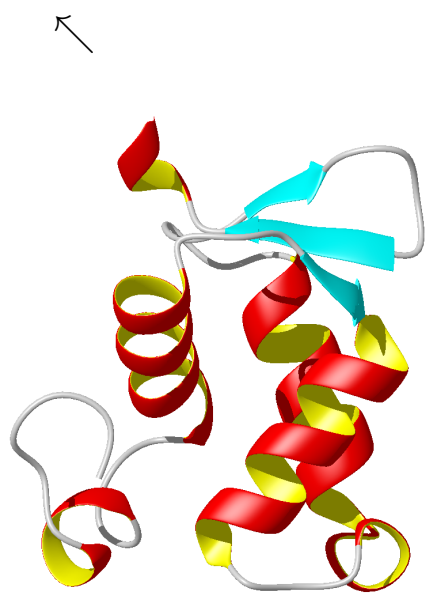

b

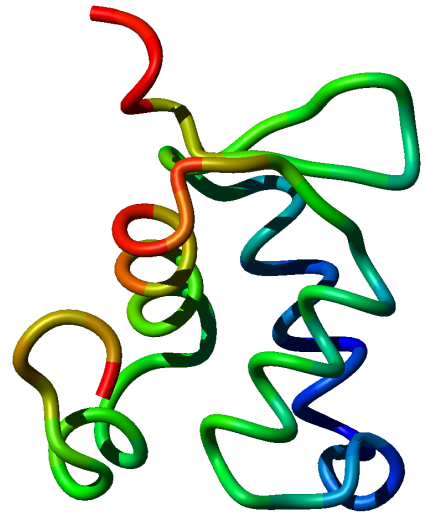

$\frac{d_{\mathrm{POOL}}-d_{\mathrm{AST}}}{d_{\mathrm{POOL}}}$

$0 \%$

$1 \%$

$2 \%$

$3 \%$

Supporting Figure S5: Regions in the N-terminal well-ordered domain of $\delta$ that show interactions with the K-tract mapped onto the known structure of the N-terminal domain (2KRC.PDB). (a) Secondary structure of the N-terminal domain. (b) Backbone of the Nterminal domain colored according to the relative distances to the K-tract (residues 96-104 in the disordered C-terminal region). Blue, green, and red colors correspond to average distances shorter by $0 \%, 1.5 \%$, and $3 \%$, in the selected ensemble $\left(d_{\mathrm{AST}}\right)$ than in the starting statistical pool of structures $\left(d_{\mathrm{POOL}}\right.$, see the scalebar). The differences, reflecting transient interactions that influenced the experimental data used for the ASTEROIDS selection, are displayed for the residue of the K-tract that exhibited the largest change in the average distance. The arrows above the structures indicates continuation of the disordered C-terminal polypeptide chain. 

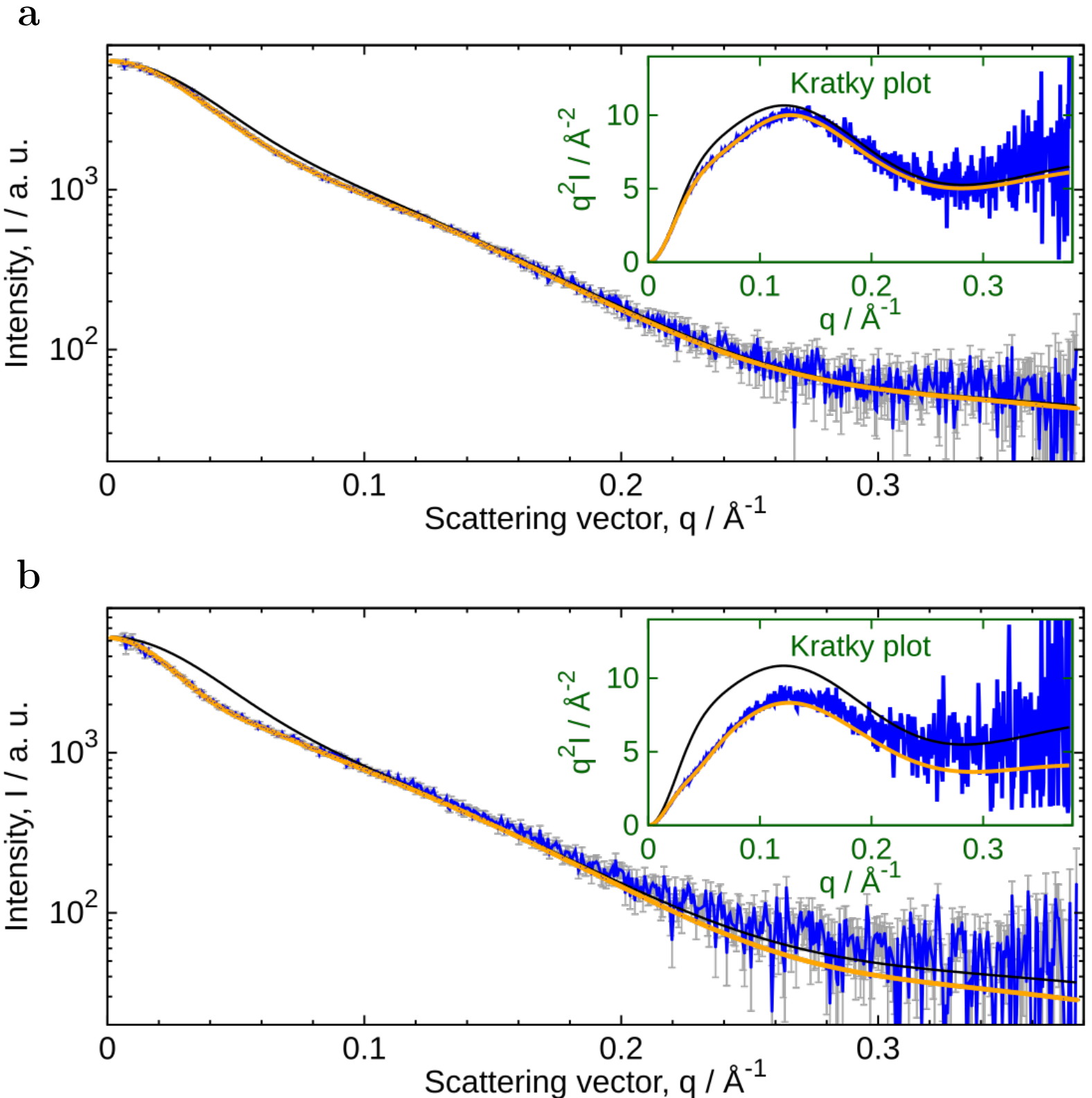

Supporting Figure S6: SAXS intensities and Kratky plots for wt $\delta$ (top) and $\delta_{\mathrm{KE}}$ (bottom). Experimental values are shown in blue, values calculated from the original statistical pool of conformers and from the ensembles selected by ASTEROIDS are shown in black and orange, respectively. 


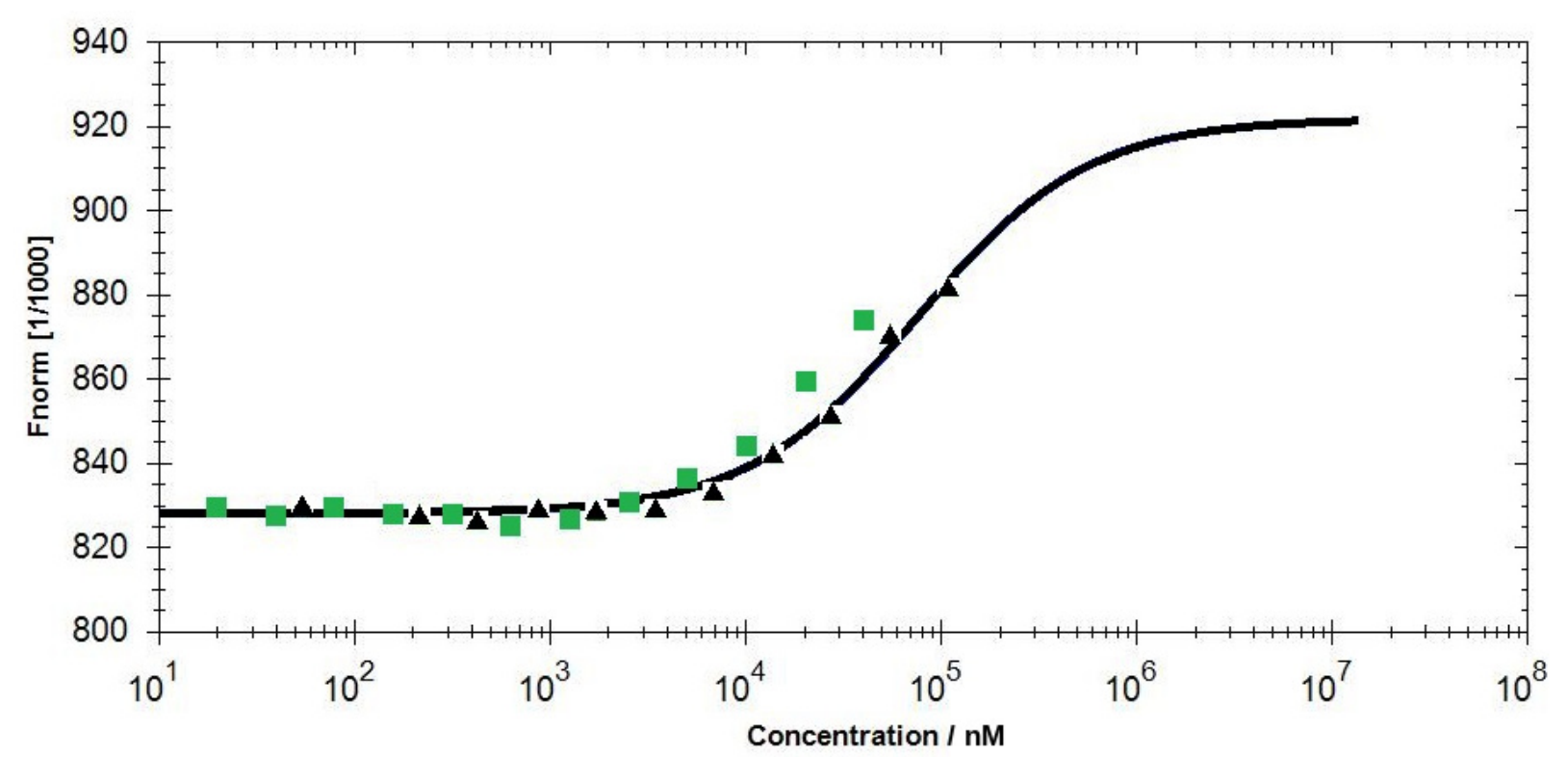

Supporting Figure S7: Microscale thermophoresis showing similar binding properties of $\delta$ (black triangles) and $\delta_{\mathrm{KE}}$ mutant (green squares) to B. subtilis RNA polymerase. Proteins have the same affinity to the RNAP core.

Microscale thermophoresis (MST) measurements were done using NanoTemper Monolith NT.115 and MST Premium Coated Capillaries (NanoTemper). All MST measurements were performed at $60 \%$ LED power and $40 \%$ MST power. MST laser on time was 30 seconds, off time was 5 seconds. RNAP $\Delta \delta$ was fluorescently labelled using Monolith His-Tag Labeling Kit RED-tris-NTA (NanoTemper) and $50 \mathrm{mM}$ Hepes pH 7.6, $150 \mathrm{mM} \mathrm{NaCl}, 5 \%$ (v/v) glycerol, $0.5 \mathrm{mM}$ DTT as the labelling buffer. Labelling was done according to the manufacturer's protocol. $50 \mathrm{mM}$ Hepes $\mathrm{pH} 7.6,150 \mathrm{mM} \mathrm{NaCl}, 5 \%$ (v/v) glycerol, $0.5 \mathrm{mM}$ DTT with the addition of $0.05 \%(\mathrm{v} / \mathrm{v})$ Pluronic F-127 non-ionic surfactant was used as the buffer in the binding experiments. Final concentration of RNAP $\Delta \delta$ in binding experiments was $33 \mathrm{nM}$ and was kept constant. For both $\delta$ and $\delta_{\mathrm{KE}}$ twelve point titration series were prepared with the dilution ration $1: 1$ with the highest concentration $\delta$ and $\delta_{\mathrm{KE}} 110000 \mathrm{nM}$ and $40500 \mathrm{nM}$, respectively. After mixing labelled $\operatorname{RNAP} \Delta \delta$ and corresponding $\delta$, mixtures were left in RT and dark for one hour in order to achieve equilibrium. 


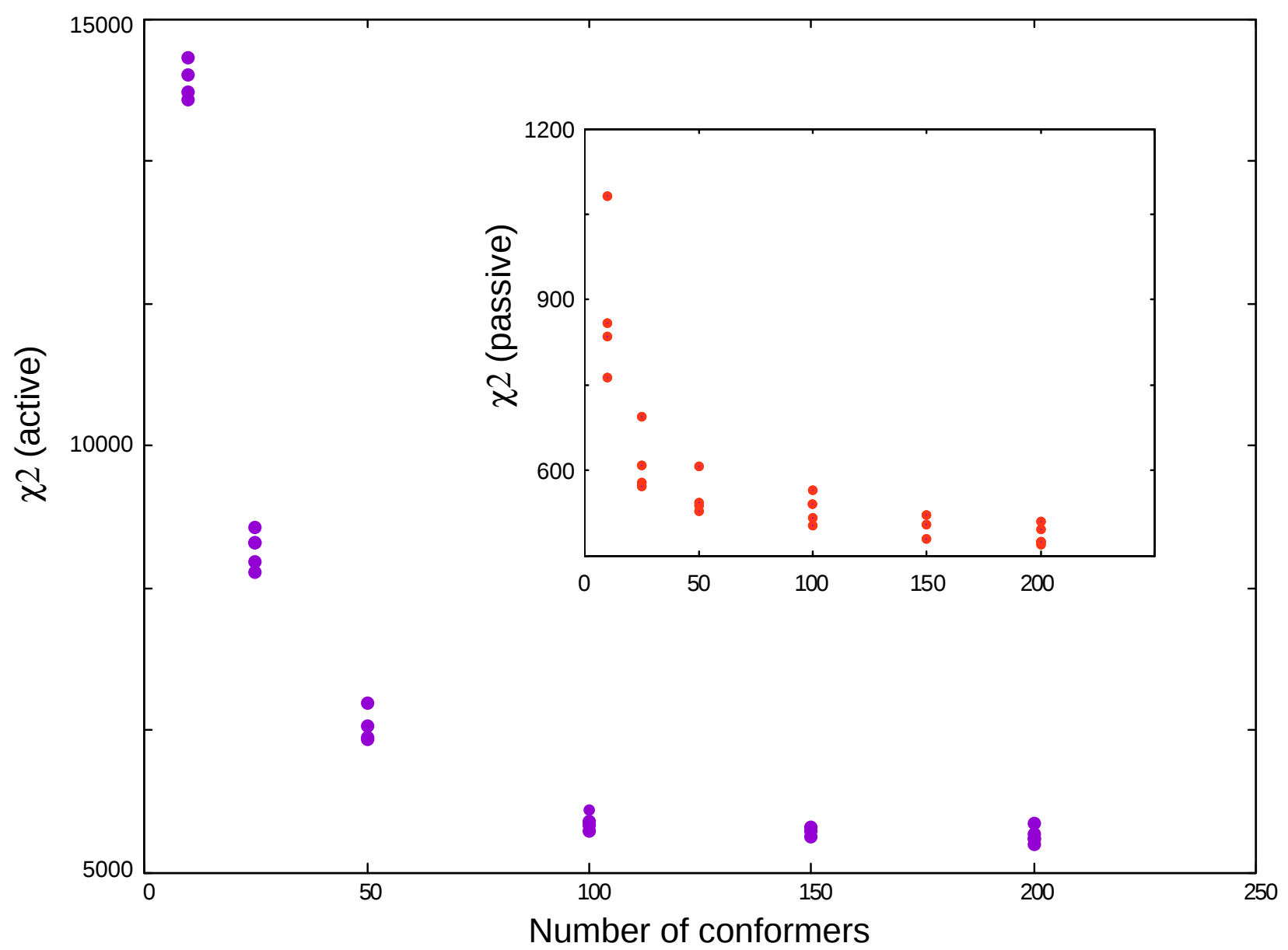

Supporting Figure S8: An evolution of $\chi^{2}$ (comprising the total $\chi^{2}$ from all experimental data) with respect to the number of conformers selected in the ASTEROIDS analysis. In addition to "active" $\chi^{2}$, an evolution of "passive" $\chi^{2}$ (calculated for $10 \%$ of all RDCs that were removed from the calculation) is presented in the inset. The behavior of the $\chi^{2}$ function is similar in both cases, and shows that 200 conformers does not appear to overfit data that are not included in the fit. 
Supporting Table S1: Sequences from the amino sequence alignment.

\begin{tabular}{ll}
\hline Sequence & Source \\
\hline Ban & Bacillus anthracis str. A2012 \\
Bce & Bacillus cereus ATCC 14579 \\
Bcl & Bacillus clausii KSM-K16 \\
Bcr & Bacillus cereus E33L \\
Bcu & Bacillus cereus subsp. cytotoxis NVH 391-98 \\
Bha & Bacillus halodurans C-125 \\
Bli & Bacillus licheniformis ATCC 14580 \\
Bst & Bacillus stearothermophilus 10 \\
Bsu & Bacillus subtilis subtilis str. 168 \\
Gka & Geobacillus kaustophilus HTA426 \\
Gst & Geobacillus stearothermophilus \\
Lla & Lactococcus lactis subsp. cremoris MG1363 \\
Lmo & Listeria monocytogenes str. 4b F2365 \\
Lwe & Listeria welshimeri serovar $6 \mathrm{~b}$ str. SLCC5334 \\
Sag & Streptococcus agalactiae A909 \\
Sau & Staphylococcus aureus subsp. aureus MRSA252 \\
Sca & Staphylococcus caprae M23864:W1 \\
Sep & Staphylococcus epidermidis \\
She & Staphylococcus haemolyticus JCSC1435 \\
Sin & Staphylococcus intermedius NCTC 11048 \\
Skl & Staphylococcus kloosi \\
Sle & Staphylococcus lentus \\
Smu & Streptococcus mutans UA159 \\
Spg & Streptococcus pyogenes MGAS8232 \\
Spn & Streptococcus pneumoniae R6 \\
Spo & Streptococcus pyogenes M1 GAS \\
Sps & Staphylococcus pseudintermedius \\
Spy & Streptococcus pyogenes str. Manfredo \\
Sth & Streptococcus thermophilus CNRZ1066 \\
Stm & Staphylococcus muscae \\
Sxy & Staphylococcus xylosus \\
&
\end{tabular}


Supporting Table S2: Proteins in the Swiss-Prot database containing the K-D/E motif.

\begin{tabular}{|c|c|}
\hline Uniprot ID & $\begin{array}{l}\text { Motif } \\
\text { Description }\end{array}$ \\
\hline \multicolumn{2}{|c|}{ Proteins interacting with nucleic acids or localized in the nucleus } \\
\hline Q93148 & $\begin{array}{l}\text { KLKKKKKVVASSDEDEDDEDDEEEGRKEMQ GFIADEDDEEEDARSEKSDRSRRSEINDEL } \\
\text { DDEDLDLIDENLDRQGERKKNRVRLGDSSD EDEPIRRSNQDDDDLQSERGSDDGDKRRGH } \\
\text { GGRGGGGYDSDSDRSEDDFIEDDGDAPRRH RKRHRGDEHIPEGAEDDARDVFGVEDFNFD } \\
\text { EFYDDDDGEEGLEDEEEEIIEDDGE } \\
\text { Suppressor of Ty } 6 \text { homolog (may regulate transcriptional elongation by } \\
\text { RNA polymerase II) }\end{array}$ \\
\hline Q54MB8 & $\begin{array}{l}\text { KKKKKKSKKNKNRHSTEEDETMEDANENLD FATGAGEEEEEEEPE } \\
\text { RNA polymerase-associated protein LEO1 }\end{array}$ \\
\hline 060841 & $\begin{array}{l}\text { KKKKDKKKKKGEKEEKEKEKKKGPSKATVK AMQEALAKLKEEEERQKREEEERIKRLEEL } \\
\text { EAKRKEEERLEQEKRERKKQKEKERKERLK KEGKLLTKSQREARARAEATLKLLQAQGVE } \\
\text { VPSKDSLPKKRPIYEDKKRKKIPQQLESKE VSESMELCAAVEVMEQGVPEKEETPPPVEP } \\
\text { EEEEDTEDAGLDDWEAMASDEETEKVEGNK VHIEVKENPEEEEEEEEEEEEDEESEEEEE } \\
\text { EE } \\
\text { Eukaryotic translation initiation factor 5B }\end{array}$ \\
\hline P30681 & $\begin{array}{l}\text { KKGKKKDPNAPKRPPSAFFLFCSENRPKIK IEHPGLSIGDTAKKLGEMWSEQSAKDKQPY } \\
\text { EQKAAKLKEKYEKDIAAYRAKGKSEAGKKG PGRPTGSKKKNEPEDEEEEEEEEEEEDDEE } \\
\text { EEEDEE } \\
\text { High mobility group protein B2 (binds DNA with a preference to non- } \\
\text { canonical DNA structures such as single-stranded DNA) }\end{array}$ \\
\hline 013741 & $\begin{array}{l}\text { KSKKKKKKLNDSSDDIEGKYFEELLAEEDE EKDKD } \\
\text { Nucleolar protein } 12 \text { RNA-binding }\end{array}$ \\
\hline Q8BTT6 & $\begin{array}{l}\text { KKQKKHLRDFGEEHPFYDRVSKKEAKPQIC QLPESSDSSHSESESESEQEHVSGYHRLLA } \\
\text { TLKNVSEEEEEEEEEEEEEEEEEEEEEEEE EDD } \\
\text { Digestive organ expansion factor homolog (binds rRNA) }\end{array}$ \\
\hline A7TJM9 & $\begin{array}{l}\text { KKGKKNDPVKINGNGDEVAEDLNPDFRFNI DGGELSTNFQGWDFVGDQKENDDDMKKDVD } \\
\text { LDGIIRRKGGLLNMAHIEVAEEEEEEEEEE EEEEEEEEEE } \\
\text { ATP-dependent RNA helicase DRS1 }\end{array}$ \\
\hline Q84MH1 & $\begin{array}{l}\text { KKKNKKKKSKKTNLKQKKAAEPKPPRDTDD DEDDEEEADDD } \\
\text { RAP domain-containing protein, chloroplastic (Oryza sativa subsp. japon- } \\
\text { ica, probable RNA-binging protein involved in chloroplast development) }\end{array}$ \\
\hline Q8VZE7 & $\begin{array}{l}\text { KKKKKKKSKKVIVKKDKVKSKSIPEDDFDT EDEDLDFED } \\
\text { RAP domain-containing protein, chloroplastic (Arabidopsis thaliana, binds } \\
\text { to } 16 \mathrm{~S} \text { rRNA precursor) }\end{array}$ \\
\hline Q26486 & $\begin{array}{l}\text { KKAKPDKKAGKNSAPAAESDSDDDDEDQLQ KFLDGEDIDTDENDESFKMNTSAEGDDSDE } \\
\text { EDDDEDEEDEEDDDEDDEEEEE } \\
\text { FK506-binding nuclear protein }\end{array}$ \\
\hline B4MR46 & $\begin{array}{l}\text { KKKKKTKKRKRKSSDDDDDESSSSDSESSS SSEEEEDEEE } \\
\text { Serrate RNA effector molecule homolog }\end{array}$ \\
\hline
\end{tabular}


Supporting Table S2: Continued.

\begin{tabular}{|c|c|}
\hline Uniprot ID & $\begin{array}{l}\text { Motif } \\
\text { Description }\end{array}$ \\
\hline Q70Z19 & $\begin{array}{l}\text { KKKPKKGSKNAKPITKTEQCESFFNFFSPP QVPEDEEDIDEDAAEELQSLMEQDYDIGST } \\
\text { IRDKIISHAVSWFTGEAAEDDFADLEDDDD DDEEDDDDEDEEEEDDEDDEDEEDEDD } \\
\text { Nucleosome assembly protein } 1 ; 1 \text { (Nicotiana tabacum) }\end{array}$ \\
\hline Q70Z18 & $\begin{array}{l}\text { KKKPKKGSKNAKPITKTEQCESFFNFFSPP QVPEDEEDIDEDAAEELQNLMEQDYDIGST } \\
\text { IRDKIIPHAVSWFTGEAAEDDYAELEDDED EDDDEEDDEDEDEEEEDEEDDEDEEEDEDE } \\
\text { Nucleosome assembly protein } 1 ; 2 \text { (Nicotiana tabacum) }\end{array}$ \\
\hline Q70Z17 & $\begin{array}{l}\text { KKKPKKGSKNSKPITKIEQCESFFNFFSPP QVPDDEEDIDEDAAEELQNLMEQDYDIGST } \\
\text { IRDKIIPHAVSWFTGEAAQDEDYIDLEDDE DEEDDEDEDEDEEDEEEEDEDEDDDDEDE } \\
\text { Nucleosome assembly protein } 1 ; 3 \text { (Nicotiana tabacum) }\end{array}$ \\
\hline Q5VND6 & $\begin{array}{l}\text { KKKPKKGSKNAKPITKTEVCESFFNFFSPP QVPDDDEDIDEDTADELQGQMEHDYDIGTT } \\
\text { IRDKIIPHAVSWFTGEAVQAEDFDDMEDDE EDDEDDDEDEEEEEEDEDEDEDDEEE } \\
\text { Nucleosome assembly protein } 1 ; 1 \text { (Oryza sativa subsp. japonica) }\end{array}$ \\
\hline Q9VNG1 & $\begin{array}{l}\text { KKLKKKKKKDEKKNLLHRQCDDTEANESDE EEEELRNEELDLEEESQMQHEELSD } \\
\text { Gametogenetin-binding protein 2-like }\end{array}$ \\
\hline A3LXX5 & $\begin{array}{l}\text { KDKKKHKKRRRRQYDDDVPKDSETKEAAED DEEEEDGEFDENNLENEEDVEDDLAEIDTA } \\
\text { NIITTGRRTRRKVIDFAKAAKELDAENGVV REDDEEEEDGEFEVKE } \\
\text { Histone H2A.Z-specific chaperone CHZ1 }\end{array}$ \\
\hline Q9U7C9 & $\begin{array}{l}\text { KKKFSQKKNHLLNLKKSYQDPEIIAHSRPR KSSGGVSLVEALSDHANYISNLDGFKYYAR } \\
\text { ANKSSLNSNATTSGGNNRSIKLNEYKYDDE EEDEEDEDEEDEEEDEEEEEEEEEEEED } \\
\text { Nucleomorphin }\end{array}$ \\
\hline Proteins o & other or uncharacterized functions \\
\hline Q6S003 & $\begin{array}{l}\text { KGKKKKKSSSPDSLSPNKDDDSSIMIDEDE EDDEEDEDDD } \\
\text { Kinesin-related protein } 8\end{array}$ \\
\hline Q23FE2 & $\begin{array}{l}\text { KPKKKKKKSKKDKQQGDTEKKEEEEGEAED EEEDEEDEEEEE } \\
\text { Tubulin glycylase } 3 \mathrm{C}\end{array}$ \\
\hline Q86IZ4 & $\begin{array}{l}\text { KDKKKKKKNLKIHMRSDSDDNDDNEDEDED ETEE } \\
\text { Protein phosphatase inhibitor } 2\end{array}$ \\
\hline Q6LFN2 & $\begin{array}{l}\text { KKKKKVKVKVKDEGKKEKIEDKKYEQDDEE ENEEEEEEEEEEEGEEENKEDEE } \\
\text { RING finger protein P01FF65c }\end{array}$ \\
\hline Q54LY8 & $\begin{array}{l}\text { KAKKKAKTEKKKDDGDEEEDEEKEEDEEQK EEQSQEEDSEESEEE } \\
\text { Uncharacterized protein DDB G0286299 }\end{array}$ \\
\hline
\end{tabular}


Supporting Table S3: Proteins in the Swiss-Prot database containing sequences similar to $\mathrm{K}-\mathrm{D} / \mathrm{E}$ motif (with shorter acidic regions or lysine repeats).

\begin{tabular}{|c|c|}
\hline Uniprot ID & $\begin{array}{l}\text { Motif } \\
\text { Description }\end{array}$ \\
\hline \multicolumn{2}{|c|}{ Proteins interacting with nucleic acids or localized in the nucleus } \\
\hline \multirow{2}{*}{ Q9HEC4 } & KKKKKKKGGDEDGSDDDEEKEE \\
\hline & $\begin{array}{l}\text { rRNA biogenesis protein rrp-36 (component of } 90 \mathrm{~S} \text { pre-ribosome involved in } \\
\text { the maturation of rRNAs) }\end{array}$ \\
\hline \multirow[t]{2}{*}{ Q9SB89 } & $\begin{array}{l}\text { KKKKKKNKKLQQRGDTNDEEDEVIAEEEEP KKKKKKQRKDTEAKSEEEEVEDKEEEKKLE } \\
\text { E }\end{array}$ \\
\hline & DEAD-box ATP-dependent RNA helicase 27 \\
\hline \multirow[t]{2}{*}{ Q9FMY7 } & KKKKKEKESYGGDPIIKDRFGGAEEEE \\
\hline & Zinc-finger homeodomain protein 13 \\
\hline \multirow[t]{2}{*}{ Q7T0Q5 } & KRKKKLKILEKLEAEGEEEEEE \\
\hline & Nucleolar protein 10 \\
\hline \multirow[t]{2}{*}{ Q6NVM6 } & KRKKKLKILEKLDAEDGEEEEEPE \\
\hline & Nucleolar protein 10 maturation of SSU-rRNA \\
\hline \multirow[t]{2}{*}{ Q6DJI9 } & KKEKKKRKRLVEEEEEEVVEEEE \\
\hline & Zinc finger CCHC-type and RNA-binding motif-containing protein 1 \\
\hline \multirow[t]{2}{*}{ Q3B7G7 } & KHKKKSKERSRGKAKKRSYSSSDEEEDE \\
\hline & Protein SREK1IP1 mRNA splicing \\
\hline \multirow[t]{2}{*}{ Q14331 } & KSKKKKSKDKKRKREEDEE \\
\hline & $\begin{array}{l}\text { Protein FRG1 (Homo sapiens, binds to mRNA, may play a role in regulation } \\
\text { of pre-mRNA splicing or in the assembly of rRNA into ribosomal subunits) }\end{array}$ \\
\hline \multirow[t]{2}{*}{ P97376 } & KSKKKKSKDKKRKREEDEE \\
\hline & Protein FRG1 (Mus musculus) \\
\hline \multirow[t]{2}{*}{ Q08211 } & KDKKKKDKDDDGGEDDD \\
\hline & ATP-dependent RNA helicase A \\
\hline \multirow[t]{2}{*}{ P41512 } & KKKKVKKEEEEKWKWWEEE \\
\hline & DNA topoisomerase 1 \\
\hline \multirow[t]{2}{*}{ P18728 } & KGKKKKKDKNEEEEEE \\
\hline & Gastrula zinc finger protein XlCGF53.1 \\
\hline \multirow[t]{2}{*}{022230} & KTKKKCKQLMELVERYRGEDEDATDESDDE EDE \\
\hline & Heat stress transcription factor B-3 \\
\hline \multirow[t]{2}{*}{ Q8TDI0 } & KKKKKKLKDKKEKKAKRKKKDEDEDDNDD \\
\hline & Chromodomain-helicase-DNA-binding protein 5 \\
\hline \multirow[t]{2}{*}{ D3ZD32 } & KKKKKKLKEKKEKKAKRKKRDEDEEDNED \\
\hline & Chromodomain-helicase-DNA-binding protein 5 \\
\hline \multirow[t]{2}{*}{ BOW0S3 } & KKKEKKKTKETKDSRKKKRVEDDDDE \\
\hline & Eukaryotic translation initiation factor 3 subunit $\mathrm{C}$ \\
\hline \multirow[t]{2}{*}{ A2QAX7 } & KQKKKKQKQEEEFSELEDDDEED \\
\hline & ATP-dependent RNA helicase drs1 \\
\hline \multirow[t]{3}{*}{ P20397 } & KKAVAKKEESEEDDDDEDESEEEKAVAKKP TPAKKPAGKKQESEEEDDEESEDEPMEVAP \\
\hline & ALKGKKTAQAAEEDDEEEDDDDEEDDDDEE E \\
\hline & Nucleolin \\
\hline
\end{tabular}


Supporting Table S3: Continued.

\begin{tabular}{cl}
\hline Uniprot ID & $\begin{array}{l}\text { Motif } \\
\text { Description }\end{array}$ \\
\hline Proteins of other or uncharacterized functions \\
Q6CTC3 & KLKKKEKEKEKKRQQQLAKEEEKKRQEEEE \\
& Stress response protein NST1 \\
Q9LVA0 & KSKKKEKTRVVVIEEEEEEDDE \\
& BAG family molecular chaperone regulator 7 \\
Q5AUG9 & KKKKKAKPGKALLSFGGDDEEDED \\
& Peptidyl-prolyl isomerase cwc27 \\
Q5AQ47 & KSKKKKKKKNKPYDDDDEEEEE \\
& NAD-dependent protein deacetylase HST1 \\
Q86IW5 & KEKKKDKQEEYIDSDDDDDDD \\
& Transmembrane protein DDB G0274347 \\
\hline
\end{tabular}

